\title{
CASE OF ABSCESS OF THE PANCREAS.
}

By JAMRS KILGOUR, M.D., Physician to the Ludlow Dispensary.

Case. T., Esq., øt. 41, unmarried, of indolent habits, a large eater, fond of sweetmeats, addicted to masturbation, and one of whose sisters was carried off by phthisis, was subject, for some years, to periodical attacks of bilious dyspepsia, usually occurring in the autumn, and often attended by a white condition of the alvine evacuation, along with a liability to sickness and vomiting, more particularly in the morning. In September 1849, he suffered as usual ; but, instead of getting well after a little dietetic discipline and laxative medicine, as had been the case in former years, his symptoms increased; and, when I first saw him, on the 21st of March last, he was emaciated, feeble, and dispirited. The symptoms under which he then laboured were sufficient to account for this condition : the most prominent were, frequent vomiting, without much nausea, great sense of distension in the stomach and bowels, which secreted much gas, almost total loss of appetite, and restlessness, with want of sleep at night. With reference to the vomiting, it usually took place in the morning, either before or after taking food; but, during the day, it generally occurred after food,-sometimes immediately, sometimes after the lapse of two or thrue hours. The matters rejected were the ingesta, mingled with, and followed by, a quantity of thin, ropy mucus, the whole being intensely acid. The amount discharged varied from a quart to an ounce, and seemed, in general, to depend upon the time during which the acid secretions had been permitted to accumulate. The act was attended with little effort; and, as has been already stated, was not accompanied by much, nor sometimes by any, nausea. The skin was dry and unperspiring, of a slightly yellow tinge, which was more pronounced in the sclerotic; the tongue white, but moist, though stated to have been red and dry previonsly; there was no thirst; the bowels were slow, but easily moved by medicine; the stools consistent, never fluid, unmixed with bile, and remarkably fetid; the urine was passed in proper quantity (sp. gr. 1.022), sometimes much loaded with lithates, and generally containing a good deal of urea and colouring matter. No bile could be detected in it; but the microscope showed it to contain fat. ${ }^{1}$ The pulse was about 100, small; no pain whatever was complained of; even firm pressure over the abdomen failed to elicit signs of tenderness at any point,- the only inconvenience occasioned by its exercise, arising from its producing a tendency to vomit. Moreover, there was no unusual prominence of its walls, either general or partial ; but percussion revealed much tympanitic resonance over the stomach and right side of the abdomen; while the left yielded a dull sound, from the lower border of the sto mach to the ilium, and felt doughy, inelastic, and, towards the median line, knotty.

The region over which this absence of resonance prevailed, had no

1 Dr. Bright mentions three cases in which fatty discharges from the bowels took place, and in which there was found, after death, a scirrhous condition of the Pancreas, and a fungous disorganisation of the duodenum. 
sharply-defined limits, nor could the form of any tumour or substance beneath the abdominal walls be traced. There was neither pulsation nor fluctuation, to aid the diagnosis; and the general impression which an examination of the abdomen, at this time, convejed, was, that some abnormal growth, occupying the greater portion of the left side of the abdomen, had pushed the intestines over to the right side, and the stomach up towards the thorax. The liver, whose secretion we have seen to be absent from the stools, though recognizable in the colour of the skin, could not be connected with this abnormal state. It appeared to be of ordinary bulk, not exceeding its natural limits in any direction, and bore pressure with the same impunity as other portions of the abdomen.

The case, as now described, proceeded, with slight and varying alterations in some of the symptoms, but with a decided downward tendency upon the whole, until about the middle of May, when its progress became accelerated by the occurrence of something like irregular intermittent. There were rigors, at uncertain intervals, of from one to three days, each being followed by a hot fit, and terminating by sweating ; there was also a tendency to sweat at night. The vomiting, from which the patient had experienced occasional and temporary relief by the use of bismuth, now returned in all its intensity, and bade defiance to remedies; the wasting and debility, which heretofore had advanced with a gradual step, became painfully apparent from day to day; the appetite grew weaker; while the pulse rose in frequency (to 114), and sank in power. The bowels, no longer slow, acted of their own accord about twice daily; the quality of their contents remaining, as already described, devoid of bile, and horribly fetid, but never fluid. The tongue became red and clean, with numerous aphthæ on its back part and edges; the urine and saliva were natural in quantity and in quality; a little thinst was complained of, but no pain. It is to be remarked of the matters romited now, that they were less sour than formerly, sometimes consisting of thin, glairy mucus, with something like yelk of egg intermixed; sometimes of a colourless fluid, neutral to test-paper, and slightly coagulable by heat. At the same time, the condition of the abdomen, as discoverable by palpation and percussion, became much changed; the dulness descending no lower than the umbilicus, and not rising higher than the ordinary situation of the lower border of the stomach, but rather stretching backward to the left flank. The cause of this dulness still remained undefinable as to form; and the abdomen, at that part, gave no other sensation to the fingers than what I have already doscribed, namely, a doughy, inelastic feel. Pressure still failed to exeite pain in any part of the abdomen; but some slight pain, or rather uneasiness, had been experienced in the left lumbar region.

The next epoch, which it is important to mark in this case, may be dated from the end of May. The rigors then ceased, and the abdomen returned to its usual condition, except at one point, about an inch above the umbilicus, and rather to the left of the mesian line, where pressure caused a feeling of soreness, or, as the patient expressed it, as if a boil were there. Over this spot, which was about two inches in diameter, the abdomen felt resistant, and India-rubber like. This apparently farourable change brought no improvement to the patient, 
because it was not accompanied by any mitigation of the mortal symptoms exhibited in the disorder of the stomach, liver, and duodenum, and the wasting consequent thereon. Neither did the pulse fall with the subsidence of the febrile fits; on the contrary, it beat 120 in the minute, and some hurry of breathing became now evident for the first time. The bowels continued to act without medicine, and a slight trace of bile was now discoverable in their contents, but their fetor remained unabated. The patient continued to be much annoyed by the quantity of gas secreted by the stomach and intestines; which, besides giving the sensation of uneasy fulness, helped to excite romiting, now become more frequent than ever, though still unaccompanied by straining or retching. As might be looked for in such circumstances, rasting and muscular debility made rapid progress; and the appearance of the patient became altered, in proportion to their advance. No noticeable change, however, took place in the principal symptoms, until about the beginning of July, when a sudden and considerable swelling of the feet and legs occurred, which was followed by much relief. The romiting became less frequent, and the appetite improved a little, but still the processes necessary for nutrition remained imperfect; bile did not find its way into the duodenum, except in very minute quantities, and, although what was eaten was not so constantly rejected as formerly, it did not contribute either to strengthen the patient, or to arrest the progress of the dépérissement which proceeded unchecked unto the end. Shortly after this, some decay in the memory and perception became obvious; there was tendency to somnolency, and the pupils occasionally dilated; the patient was sunk down in bed, and declined the least exertion; the features were pinched, and cadaveric, and it became apparent that the cessation of the severer symptoms was only an evidence of the waning powers of life. On the evening of the 26th, the vegetable-like monotony of the patient's existence was broken, by the occurrence of a severe rigor, from which he recovered, to experience a second on the following evening, and a third on the morning of the 28th, which last extinguished the feeble and flickering flame of life.

The post-mortem examination was made fifty-eight hours after death, when decomposition had already made some progress, and, from circumstances, in a very hurried manner. The limbs were extremely emaciated, the trunk much less so, and half an inch of fat was found to cover the muscles of the abdomen, which alone was opened. Much fetid gas escaped upon puncturing its cavity, and some fat was found in front of the intestines. The stomach was very large and distended with gas, its walls were extremely thin, and some inflammatory patches were observed near the pylorus, on the lesser curvature. The pylorus itself was slightly thickened, and its orifice narrowed. The duodenum bore the marks of intense inflammation throughout, which had proved most destructive in that portion most contiguous to the Pancreas, which broke down into pultaceous shreds in the most cautious attempts to examine it. The inflammation extended for a short distance into the jejunum, and there stopped. The liver was slate-coloured, of ordinary bulk, and normal texture. The gall-bladder was healthy, and contained about three teaspoonfuls of orange-coloured bile. The ducts were all pervious. The spleen was not less than one-third larger than usual 
but healthy. The Pancreas; with its envelopes greatly thickened, formed a mass whose diameter would somewhat exceed that of a large breakfast cup. It adhered to the left supra-renal capsule, but to no other of the adjoining riscera. When cut into, a creamy pus flowed out; and, upon further investigation, the entire gland was found to be converted into a cyst, filled with the pus just mentioned, and a few cheesy masses about the size of a bean. ${ }^{\prime}$ Remains of septa in the cyst were visible, from which it nould seem that the suppurative process was first completed in one portion of the organ, and then in another. The kidneys were healthy; only that part of the left kidney in con. nexion with the diseased Pancreas was red. I had no opportunity of examining the contents of the cyst, nor any part of the diseased structures, with the microscope.

Reyarks. In remarking upon the above case, which is not one of common occurrence, ${ }^{2}$ the most obvious features which strike the eye are the vomiting and wasting, which commenced at an early period of the disease and accompanied it to its close. The absence of pain is also remarkable; and the more so, as it was certain from the physical signs, that the various phenomena, which the case presented, were not altogether dependent upon functional derangement of the abdominal viscera. Again, the immunity of the nervous system and integrity of many of the secretions, adds one to the occasional proofs which are afforded to us, of the great extent to which the destruction even of an important organ may go, without compromising any other system than the one immediately concerned. The pulse was increasingly quick throughout, but its acceleration did not seem to depend upon any particular phase of the complaint, but rather on the increasing debility of the patient.

Most writers who have treated of Pancreatic disease have admitted the frequency of vomiting, ${ }^{3}$ and some have considered it an essential symptom. Morgagni points out how Disease of the Pancreas may produce vomiting; but, in his third book, he says, "I even confess that this viscus has been found diseased by me and by others without a vomiting being the consequence of it." Dr. Abercrombie inclines to the same opinion, as does also Dr. Claessen of Cologne, who has reported upon 322 cases of Disease of the Pancreas. Indeed it seems reasonable to suppose, that in many cases of this nature, particularly when the morbid change has not proceeded far, vomiting should not occur as a constant symp-

1 According to Gendrin, in large purulent deposits in the Pancreas, the pus has generally been found creamy and inodorous ; Portal, on the other hand, states, that in complete suppuration of the Pancreas, the pus is sometimes of an intolerable smell, and in very large quantity, amounting to two pounds or upwards. The same authority speaks of such abscesses as occasionally the result of disease of the testicle ; and M. Tonelle mentions two cases of puerperal peritonitis, attended with like consequences.

Among the 322 cases of Diseased Pancreas recorded by Dr. Clacesen, thert is not one case of abscess.

8 The fact of large quantities of watery fluid being vomited, when the Pancreas has been found diseased, led to an opinion, at one time generally entertained, that the fluid rejected consisted principally of the secretion of that organ. This idea later writers have held to be incorrect, and have argued that the stomach is the source of the secretion, an opinion which this case puts beyond doubt, an it is certain that, long before denth, the Pancreas ceased to be a secreting organ. 
tom, inasmuch as neither the mochanical nor other condition for producing this effect, may exist in the required degree. In cases where obstinate vomiting does occur, where that vomiting has principally, or partly, a mechanical character, and where it can be made out that an enlarged liver or spleen is not the exciting canse; further; when the matters rejected are thin, ropy, sour, tasteless, or salty, and in considerable quantities; and when these conditions exist with pain, hardness, or tumefaction at the situation of the Pancreas, we should then, I conceive, be warranted in setting down the Pancreas as the fons et origo mali. Wasting, also, has been much insisted on, particularly by Dr. Pemberton, as indicative of Pancreatic disease. The record of this case agrees with that opinion, but I should be inclined to modify it generally upon the same principles which I have assumed as likely to determine vomiting. For instance, in cases when the functions of the stomach, liver, and duodenum escape the profound alteration which this case shows they sometimes undergo, I should not be prepared to expect a rapid emaciation; and in this view $I$ am borne out by the reports of some of Dr. Abercrombie's cases, where the bodies were found after death to contain much fat.

This question, however, involves another, viz., the extent to which the Pancreatic juice is necessary to nutrition. The experiments of M. Bernard have tended to shew, that its function is to digest the fatty matters, contained in the food, and facilitate their absorption; but the confirmation, or disproval of this opinion will rest with the statistics of Pancreatic disease. It would seem, then, that we can hardly estimate the value of emaciation, per $8 \varepsilon$, as a diagnostic mark of this complaint; but, in conjunction with the other signs enumerated as collectively presumptive of its presence, it becomes significant and valuable. Jaundice has often been found to accompany an abnormal condition of the Pancreas, which state may be presumed to influence the liver as it does the stomach, both mechanically and sympathetically. That it acts in the latter way is probable, from the experiments performed by Brunner upon dogs, from which he cut out a portion of the pancreas, and so produced death by bilious romiting. In this instance, the jaundice was slight, and dependent upon the more or less complete arrest of the flow of bile into the duodenum, yet neither diarrhoa nor obstinate constipation, one or the other of which is stated generally to accompany this disorder, occurred. A very fetid condition of the stools existed throughout, which may probably be assigned to the absence of bile as a chief cause ; yet I do not recollect to have ever observed the white evacuations of jaundiced subjects so offensive. Pain is much insisted on by Dr. Claessen as evidence of this disorder, but we have seen that this case forms an exception to his rule. The secretion of the salivary glands, which has been represented as vicarious of that of the Pancreas, when the latter has become suppressed, was not affected ; and the circulation, which has been usually stated as seldom implicated in the general disorder, was much quickened on this occasion, its excitement being apparently proportional to the duration of the disease.

Before concluding this resumé, it may be worth while to speculate for a moment upon the probable programme of the morbid actions, which at length converted the Pancreas into a bag of pus. Keeping in mind the previous history of the patient and his babits, and particularly the facts that white stools, and vomiting chiefly in the morning, gener- 
ally accompanied his anuual dyspeptic visitation, we are rather led to conjecture that an eulargement of the Pancreas had existed for some years, probably dependent upon, or connected with, a low kind of inflammation; that disturbances of the other viscera, such as usually occur in the autumn, roused the slumbering fire into temporary activity, and each succeeding year with increasing effect; that the habits of the patient, favouring the morbid tendency, prevented a complete, and only permitted an apparent recovery, and that thus the way was paved for the last final effort of the destructive process. If further, we should try to connect that process with the physical signs which furnished so important an aid to the diagnosis, I should be inclined to presume, that an extensive inflammatory swelling of the Pancreas took place, as wo see to happen to the parotid in mumps, by which the intestines became displaced, and dulness upon percussion resulted. That then this swelling subsided, upon the occurrence of suppuration, as marked by the first series of rigors reported, and the process lay dormant for a season, again finally resuming its activity, to become completed almost with the last breath of the sufferer.

It seems strange, that so decided an inflammatory action, as the necropsy gave us the evidence of, should have been unaccompanied by pain. I can only endeavour to account for it upon the supposition, that the inflammation was of a scrofulous nature, and the temperament of the patient sluggish and phlegmatic. This, however, can only be accepted as a partial explanation of a very curious fact in the pathology of this class of complaints.

I shall conclude this case by mentioning a fact which seems to possess some interest in connexion with it. Since the date of the matters here referred to, I have been consulted by a gentleman, aged 30 , of active habits, a first cousin of the deceased, for a deep seated pain felt at the situation of the Pancreas, which is neither constant nor periodic, but somewhat capricious in its onset, and apparently uninfluenced by any plan of medical treatment or diet heretofore adopted. It is represented as being of a dull aching character, and is rather relieved by light, but aggravated by heavy pressure. It sometimes changes its situation to a small extent, but not for any length of time. It has occurred on several occasions, lasting from three to six weeks during spring or autumn. It has not been accompanied by any dyspeptic symptom, but seems to stand alone, producing no effect but that arising from the sensation of pain. Here, perhaps, we have a first indication of pancreatic disease, and if so, we may learn that a derangement of its organism, sufficient to produce a tolerably severe pain, may not givo rise to any recognisable derangement of its functions, nor to any sympathetic disturbance of adjoining organs.

Iudlow, October 1850. 